\title{
Sleep-Dependent Plasticity Requires Cortical Activity
}

\author{
Sushil K. Jha, ${ }^{1 \star}$ Brian E. Jones, ${ }^{1 \star}$ Tammi Coleman, ${ }^{1}$ Nick Steinmetz, ${ }^{1}$ Chi-Tat Law, ${ }^{1}$ Gerald Griffin, ${ }^{1}$ Josh Hawk, ${ }^{1}$ \\ Nooreen Dabbish, ${ }^{1}$ Valery A. Kalatsky, ${ }^{2}$ and Marcos G. Frank ${ }^{1}$ \\ ${ }^{1}$ Department of Neuroscience, School of Medicine, University of Pennsylvania, Philadelphia, Pennsylvania 19104-6074, and ${ }^{2}$ Department of Electrical and \\ Computer Engineering, University of Houston, Houston, Texas 77204-4005
}

Recent findings in humans and animals suggest that sleep promotes synaptic plasticity, but the underlying mechanisms have not been identified. We have demonstrated recently an important role for sleep in ocular dominance (OD) plasticity, a classic form of in vivo cortical remodeling triggered by monocular deprivation (MD) during a critical period of development. The mechanisms responsible for the effects of sleep on OD plasticity are unknown but may depend on neuronal activity in the sleeping brain. We investigated the role of cortical activity in sleep-dependent plasticity by reversibly inactivating the sleeping visual cortex (V1) after a period of MD. Critical period cats were bilaterally implanted with cannulas in V1 and standard EEG/EMG electrodes for polysomnographic recording. After a period of $\mathrm{MD}$, visual cortices were infused with the sodium channel blocker lidocaine in vehicle or vehicle only during sleep. A third group of cats served as sham controls and were infused with lidocaine outside of V1 (into the CSF). Both optical imaging of intrinsic cortical signals and microelectrode recordings showed that OD plasticity was significantly reduced in cats whose visual cortices were reversibly silenced during sleep. These findings demonstrate that the mechanisms governing this form of sleep-dependent plasticity require cortical activity. They provide an important insight into how sleep modifies synaptic circuitry by narrowing the range of possible candidate mechanisms to those that are activity dependent.

Key words: development; NREM; synaptic remodeling; activity-dependent; ocular dominance; visual cortex; critical period

\section{Introduction}

Converging lines of evidence strongly suggest that sleep plays an important role in synaptic plasticity. Increasingly sophisticated studies in humans and animals show that sleep facilitates processes thought to depend on synaptic remodeling, such as learning and memory (Datta et al., 2004; Walker and Stickgold, 2004). The beneficial effects of sleep on human memory are associated with a reactivation of brain areas engaged in the learning task (Laureys et al., 2001; Huber et al., 2004). Similar reactivation on the level of single neurons and neuronal ensembles has been observed during sleep in nonhuman vertebrates, suggesting that information acquired during wake is reprocessed in subsequent sleep (Wilson and McNaughton, 1994; Nadasky et al., 1999; Louie and Wilson, 2001; Hoffman and McNaughton, 2002). Molecular studies have also identified several plasticity-related genes that are specifically upregulated during normal sleep and in sleep after long-term potentiation (Ribeiro et al., 2002; Cirelli et al., 2004). Nevertheless, the idea that sleep promotes brain plasticity remains controversial (Vertes and Eastman, 2000; Siegel, 2001). There are relatively few studies that show direct effects of sleep on synaptic

\footnotetext{
Received July 1, 2005; revised Aug. 25, 2005; accepted Aug. 26, 2005.

This work was supported by departmental funds from the University of Pennsylvania and United States Public Health Service Grant 5-R01-MH067568. We thank Joel Benington for comments on this manuscript, Naoum Issa for advice on image processing, and Amanda Codd, Terry Dean, and Sami Saba for assistance with experimental procedures

*S.K.J. and B.E.J. contributed equally to this work.

Correspondence should be addressed to Dr. Marcos G. Frank, Department of Neuroscience, School of Medicine, University of Pennsylvania, Philadelphia, PA 19104. E-mail:mgf@mail.med.upenn.edu.

DOI:10.1523/JNEUROSCI.2722-05.2005

Copyright $\odot 2005$ Society for Neuroscience $\quad 0270-6474 / 05 / 259266-09 \$ 15.00 / 0$
}

plasticity, and, although the changes in gene expression and neural activity observed during sleep are quite intriguing, their functional significance is unknown (Benington and Frank, 2003).

We have shown previously that sleep and sleep loss directly influence a well described form of in vivo cortical plasticity (Frank et al., 2001). During a critical period of development, monocular deprivation (MD) initiates a rapid remodeling of synaptic weights in primary visual cortex (V1) in favor of the open eye (Hubel and Wiesel, 1970). Short periods of sleep enhance the effects of MD, and this enhancement does not occur in the absence of sleep (Frank et al., 2001) or in other brain states [e.g., anesthesia (Freeman, 1979; Imamura and Kasamatsu, 1991)]. The underlying mechanisms responsible for this form of sleepdependent plasticity have not been identified but may depend on cortical activity. Complete blockade of cortical activity with tetrodotoxin inhibits ocular dominance (OD) plasticity (Reiter et al., 1986), and neuronal activity during sleep may facilitate synaptic remodeling (Wilson and McNaughton, 1994; Steriade and Timofeev, 2003). In this investigation, we considered the role of cortical activity in sleep-dependent plasticity by reversibly inactivating the sleeping V1 after MD. This was accomplished by temporary inhibition of neuronal activity in $\mathrm{V} 1 \mathrm{using}$ lidocaine, a sodium channel blocker previously used in studies of the visual system (Malpeli, 1999).

Parts of this paper have been published previously in abstract form (Jones et al., 2004).

\section{Materials and Methods}

Experimental procedures: main groups. To determine whether sleepdependent plasticity requires cortical activity, we reversibly inactivated 
V1 with lidocaine in sleeping cats after a brief period of MD shown previously to induce cortical remodeling (Frank et al., 2001). Cats at the peak of the critical period for OD plasticity were divided into three main (and one supplemental, see below) groups: $\mathrm{MD}+$ sleep + lidocaine (LIDO) $(n=6), \mathrm{MD}+$ sleep + vehicle (VEH) $(n=7)$, and MD + sleep + sham infusion (SHAM) $(n=6)$ groups. After postoperative recovery from the implantation of EEG/EMG electrodes and cannulas placed bilaterally either within V1 (LIDO and VEH) or outside V1 (SHAM), all cats underwent the following procedures adapted from our previous study (Frank et al., 2001). First, 6 h of baseline sleep/wake data were collected to verify that all of the cats were similar with respect to sleep/wake development and previous sleep/wake history. The cats were then briefly anesthetized with isofluorane, and the right eyelids were treated with a local anesthetic (topical lidocaine jelly) and sutured shut. After recovery, the cats were then kept awake for $6 \mathrm{~h}$ to provide a common stimulus for synaptic remodeling (MD period) as described previously (Frank et al., 2001). After the completion of the MD period, LIDO, $\mathrm{VEH}$, and SHAM cats were allowed to sleep ad libitum in complete darkness for an additional $6 \mathrm{~h}$ (post-MD period). During the ad libitum sleep period, V1 was continuously infused $(0.3 \mu \mathrm{l} / \mathrm{min})$ with either lidocaine ( $2 \%$ in vehicle; LIDO group) or vehicle only [artificial CSF (Harvard Apparatus, Holliston, MA); VEH group]. SHAM cats were treated identically as the LIDO cats, except that the cannulas were positioned outside of V1 (0.5-1 $\mathrm{mm}$ above the pial surface). After the ad libitum sleep period, all cats were immediately anesthetized to ensure that the recovery from lidocaine occurred under conditions in which further remodeling was blocked [anesthesia inhibits ongoing plasticity in V1 (Freeman, 1979; Imamura and Kasamatsu, 1991)]. The cats were then prepared for optical imaging of intrinsic cortical signals and single-unit electrophysiology (see below). Although critical period cats are aperiodic with respect to sleep/wake organization (Laguzzi et al., 1979), all experiments began such that optical imaging and electrophysiology began during the light phase. For example, baselines were recorded at $\sim 11: 00-11: 30$ A.M. to 5:00-5:30 P.M., MD periods began at 6:00 P.M. and ended at 12:00 A.M., and animals were prepared for imaging at 6:00 A.M. Rescue experiments began 3-4 h earlier so that imaging and microelectrode recordings were conducted at approximately the same times as the other groups (for a schematic representation of the experimental design, see supplemental Fig. 1, available at www.jneurosci.org as supplemental material).

Experimental procedures: supplemental groups. We also determined whether additional sleep after the initial lidocaine infusion might reverse the effects of cortical inactivation during sleep. This was accomplished by preparing two additional critical period cats (from different litters) for polysomnographic recording and V1 infusion as described above. These RESCUE cats were then treated identically as the LIDO cats, except that an additional $6 \mathrm{~h}$ ad libitum sleep period was provided after the initial sleep + infusion period. During this time, the cats remained in complete darkness with the infusion pump turned off. They were then immediately prepared for optical imaging and electrophysiological recordings as described below.

Housing conditions and formation of groups. Critical period cats from our colony were randomly assigned to each group as they became available. Before use in an experiment, kittens were housed with their queens on a $12 \mathrm{~h}$ light/dark (LD) cycle at an ambient temperature of $22^{\circ} \mathrm{C}$ and were provided food and water ad libitum. To control for potential litter effects, no more than two to three kittens from any one litter were used in any one group. Surgeries were performed so that, after postoperative recovery, all of the cats were at similar ages at the start of each experiment (mean \pm SEMs postnatal age in days: LIDO, $32.8 \pm 1.5$; VEH, $32.6 \pm$ 0.86; SHAM, $34.4 \pm 1.4$; RESCUE, $31.3 \pm 1$ ). All other procedures were as described previously (Frank et al., 2001).

Surgical procedures and sleep/wake recording. At postnatal days 24-P28, cats were anesthetized and prepared for chronic implant surgery according to previously described methods (Frank et al., 2001). The skull was exposed, five to six EEG electrodes were placed bilaterally in frontal and parietal bone (1-1.5 cm distant from V1), and three EMG electrodes were placed in the nuchal muscle. The electrodes were connected to an electrical socket that was fixed to the skull with bone screws and dental acrylic. A $1.5 \times 1.5 \mathrm{~mm}$ opening was then made bilaterally over V1, the dura was partially reflected, and a Plastics One (Roanoke, VA) dual cannula/electrode was lowered into V1 in VEH, LIDO, and RESCUE cats (0.2-0.5 mm lateral to medial bank). The internal "dummy" cannula was positioned so that it slightly perforated the pia to a depth of $0.3-0.4 \mathrm{~mm}$; in this way, a small well in the cortical tissue was made for the injection cannula used during the infusion. The assembly was fixed to the skull with bone screws and dental acrylic. After $4-5 \mathrm{~d}$ of postoperative recovery, the cats were placed in an illuminated sleep-recording chamber (22$24^{\circ} \mathrm{C}$ ) with a revolving base. The cats were provided food and water $\mathrm{ad}$ libitum at all times except during the MD. LD schedules and ambient temperature were identical to colony housing with the exception that ad libitum sleep periods always began in the dark phase.

Polygraphic signals were routed from the animal via an electrical, counter-balanced tether/commutator to an Astro-Med (West Warwick, RI) amplifier system, processed with a high-pass filter of $0.3 \mathrm{~Hz}$ and a low-pass filter of $100 \mathrm{~Hz}$, digitized at $200 \mathrm{~Hz}$, and collected on a personal computer running commercial sleep acquisition/analyses software (Kissei Comtec, Irvine, CA). Fourier-transformed EEGs from sites at the infusion site and distant from the infusion site were then averaged into delta $(0.5-4.0 \mathrm{~Hz})$, sigma $(10-15 \mathrm{~Hz})$, and high-frequency (HF) (15-40 $\mathrm{Hz}$ ) bands and normalized to mean baseline values for quantitative assessment of EEG changes during the MD and infusion periods. EEGs from the distant site (not affected by infusions) were also used to assign the states of rapid eye movement (REM) sleep, non-REM (NREM) sleep, and wake in $8 \mathrm{~s}$ epochs. Vigilance states were determined using standard polysomnographic techniques as described previously (Frank et al., 2001), and the amount of REM sleep, NREM sleep, and wake was computed for the baseline, MD, and post-MD periods.

Infusion procedures. We adapted procedures used previously to reversibly inactivate neurons in the visual system (Hupe et al., 1999; Malpeli, 1999). Previous studies have shown that infusion rates comparable with the ones used in this study minimally damage surrounding tissue and produce completely reversible suppression of neuronal activity that extends in a sphere $\sim 2-3 \mathrm{~mm}$ in diameter from the cannula tip (Hupe et al., 1999; Malpeli, 1999). We verified that similar reversible inactivation occurred in our design by optically imaging V1 in pilot studies before and after lidocaine infusion (supplemental Fig. 2, available at www.jneurosci.org as supplemental material).

Optical imaging. Optical imaging was conducted according to previously described methods (Frank et al., 2001; Kalatsky and Stryker, 2003). Briefly, a craniotomy was made, and the dura was reflected in anesthetized, paralyzed cats (isoflorane followed by Nembutal with Flaxidil or pancuronium bromide). After allowing sufficient time for full recovery from lidocaine $(2-3 \mathrm{~h})$, contact lenses were placed in the eyes (for optimum focus to a monitor positioned at $40 \mathrm{~cm}$ ), and a CCD camera was focused 500-600 $\mu \mathrm{m}$ beneath the pial surface. The cortex was illuminated with green (for vascular maps) or red (for intrinsic signals) light through the use of interference filters $(546 \pm 10$ and $610 \pm 10 \mathrm{~nm}$, respectively). A second red filter was also interposed between the camera and the cortical surface before intrinsic signal imaging. Care was taken to ensure that, in every case, the cannula site was identified and the camera focused within 1-3 mm of the cannula site [which was an area optimally inactivated by lidocaine (supplemental Fig. 2, available at www.jneurosci.org as supplemental material)]. Occasionally, the surgical placement of the cannula and EEG electrodes in V1 caused extensive pial damage or vascularization 1-3 mm near the injection site. These hemispheres were always excluded from optical analyses but, if sufficiently undamaged, were used for microelectrode recording. In some cases, we also obtained optical maps (in hemispheres ipsilateral to the deprived eye) far from the infusion site ( $>3$ $\mathrm{mm}$ ) in LIDO cats for later comparison with maps near the infusion site.

Images of the cortical surface were continuously acquired by a Dalstar $1 \mathrm{M} 30$ CCD camera (Dalsa, Waterloo, Ontario, Canada) at a rate of 30 frames/s and saved to a personal computer after temporal (four frames) and spatial $(2 \times 2$ pixels $)$ binning. The images were acquired at 2.7 magnification using a $135 \mathrm{~mm} / 50 \mathrm{~mm}$ lens combination while a continuously rotating, full-field grating was presented to either the reopened deprived eye or the nondeprived eye $\left(0.2\right.$ cycles $/{ }^{\circ}, 2$ cycles $/ \mathrm{s}$, one full rotation per minute). Single 10-12 min presentations were made to each eye in an alternate manner (e.g., clockwise rotation deprived eye, clock- 
wise rotation nondeprived eye; counterclockwise rotation deprived eye, counterclockwise rotation nondeprived eye). Altering the order of presentations had no effect on map quality. We continuously monitored the physiological state of each cat during imaging to ensure that heart rate and expired $\mathrm{CO}_{2}$ levels were always comparable between left eye and right eye presentations. We also examined a number of representative pixel responses in every map (as described by Kalatsky et al., 2003) to verify that the mapping signal was not obscured by vasomotor, respiratory, or other potential noise sources.

Optical maps were constructed as described by Kalatsky and Stryker (2003). Briefly, the slow variation in the optical signal was removed by a uniform temporal filter (120 s), and the second Fourier component, which represents the orientation parameter space, was used to generate all maps. The calculated Fourier components were then divided by the average of all frames to reduce effects of uneven illumination, and the resulting maps were minimally processed with a high-pass spatial filter (uniform circular kernel of 120-140 pixels, 1.0-1.3 mm). A low-pass spatial filter (uniform circular kernel of $8-12$ pixels, $70-100 \mu \mathrm{m}$ ) was used for display purposes but was never used in OD assessments. After correction for hemodynamic delay as described by Kalatsky et al. (2003), optical maps generated for each eye were cropped to remove vascular artifacts. Stimulus-induced luminance changes at each pixel were then used to create angle maps, polar maps, and OD ratio maps (the ratio of nondeprived/deprived eye responses at each pixel). Changes in OD were quantified using an optical contralateral bias index (CBI) and monocularity index (MI) similar to measurements used previously to analyze optical signals (Issa et al., 1999; Frank et al., 2001). Briefly, this involved computing an ipsilateral/contralateral eye ocular dominance ratio at each pixel $[\mathrm{OD}$ ratio $=\mathrm{I} /(\mathrm{C}+\mathrm{I})$, where $\mathrm{I}$ is ipsilateral response and $\mathrm{C}$ is contralateral response] and then calculating weighted averages of the pixel ratio distributions (collapsed into seven bins). These are essentially the same calculations used to generate unit CBIs and MIs, which are also weighted averages of data binned into histograms (i.e., OD ranks of 1-7). The optical MI measures how monocular the population response is and varies from 0 to 1 , with 1 indicating a completely monocular distribution of pixel responses. The optical CBI measures the degree to which pixels in a sampled area are dominated by the contralateral eye and varies from 0 to 1 , with 1 indicating complete dominance by the contralateral eye. As was done for single-unit measurements (see below), this calculation was adjusted for combined hemisphere data so that scores of 1 would indicate complete dominance by the nondeprived eye.

Microelectrode recordings. After optical imaging, a $1 \times 1 \mathrm{~mm}$ matrix of 16 electrodes (Frederick Haer Company, Bowdoinham, ME) was positioned within 1-2 $\mathrm{mm}$ of the infusing cannula and advanced in five to eight $100 \mu \mathrm{m}$ steps from the pial surface. Two or three penetrations per hemisphere were made to ensure that different cortical regions within the infusion area were sampled. Eight full-field, slowly drifting, reversing gratings $\left(0.2 \mathrm{cycles} /{ }^{\circ}, 5 \mathrm{~s}\right.$ presentation $)$ were randomly presented to each eye four times $\left(4 \times 8\right.$ different orientations at $22.5^{\circ}$ intervals plus blank screen per eye), and single units were sorted off-line using principal component analysis (OfflineSorter; Plexon, Dallas, TX). Approximately two to four neurons could be sorted per electrode, and we were typically able to record units on 12-14 electrodes in any given experiment. On average, we isolated 75-125 neurons per penetration. Mean response properties for all stimuli were calculated for each sorted neuron by a computer program. This allowed for completely objective (blind to the experimental condition) assessments of neuronal responses. We then calculated the following measures for each hemisphere, and all hemisphere values were averaged for each experimental condition.

Visual responsiveness (VR) is calculated by the equation $\mathrm{VR}=R_{\text {spon }} /$ $R_{\mathrm{pf}}$, where $R_{\text {spon }}$ is the mean number of spikes during blank screen (no stimulus) presentations, and $R_{\mathrm{pf}}$ is the mean number of spikes at the preferred orientation (maximal response). The VR ranges from 0 (a very responsive neuron, i.e., no blank screen responses) to $\geq 1$ (a nonresponsive neuron, i.e., neuron fires equally or more to blank screen as to visual stimuli). Neurons with VR values $\geq 1$ were automatically classified as nonresponsive.

For ocular dominance, we used a modified OD ranking system originally developed by Hubel and Weisel (1970). OD scores were automati-

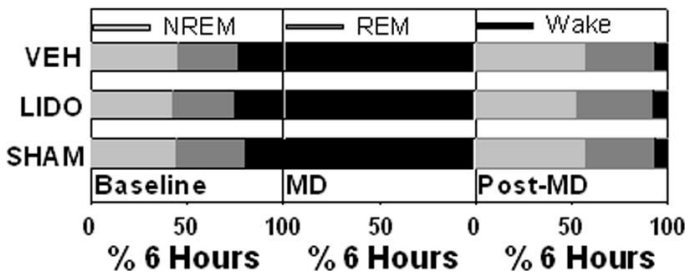

Figure 1. Vigilance state amounts in the main groups during the baseline, MD, and post-MD periods. Data represent mean state amounts expressed as a percentage of each $6 \mathrm{~h}$ interval (LID0, $n=6$; VEH, $n=7 ;$ SHAM, $n=6$ ). There were no significant differences in vigilance state amounts between the groups in any interval.

cally calculated using rules that simulated the subjective scoring of computerized spike records, or auditory assessments of neuronal responses traditionally used in the past (Hubel and Wiesel, 1970; Trachtenberg et al., 2000; Frank et al., 2001). For combined data from all hemispheres, OD scores of 7 and 1 were assigned when a neuron was dominated by the right (deprived) eye or left (nondeprived) eye, respectively. For example, scores of 1 were assigned if deprived eye responses were less than or equal to background firing (blank screen presentation). An OD score of 4 was assigned to neurons driven equally by the two eyes $( \pm 25 \%$ difference in spike rates). An OD score of 8 was assigned to neurons that were not visually responsive (see above). Intermediate scores of 2, 3 and 5, 6 were, respectively, assigned when the response to one eye was $26-200 \%$ larger (scores of 3 and 5 ) or $>200 \%$ larger (scores of 2 and 6). We also ranked unit recordings in each hemisphere using a traditional scheme in which scores of 1 and 7 indicate complete dominance by the contralateral or ipsilateral eye, respectively (Frank et al., 2001). After the assignment of OD scores, scalar measures of eye dominance (CBI and MI) were computed for all neurons in a given experimental condition (per hemisphere) according to previously described methods (Issa et al., 1999).

We verified that our algorithm approximated subjective scoring rules by examining OD histograms from six normally sighted critical period cats (supplemental Fig. 3, available at www.jneurosci.org as supplemental material). Previous studies using conventional, subjective scoring of neuronal responses show that neurons from normally sighted critical period cats are predominantly binocular (i.e., receive scores of 3,4 , and 5) (Hubel and Wiesel, 1970; Crair et al., 1998; Trachtenberg et al., 2000). The corresponding CBI and MI scores in normally sighted cats are $\sim 0.51-0.55$ and 0.30-0.35 (Reiter et al., 1986; Crair et al., 1998; Trachtenberg et al., 2000). Our algorithm generated similar binocular OD histograms with comparable scalar measures of contralateral eye bias and monocularity (mean \pm SEMs; CBIs and MIs, $0.51 \pm 0.03$ and $0.37 \pm$ 0.02 , respectively).

Statistics. Both parametric and nonparametric statistics have been used to evaluate scalar measurements of OD (Gordon and Stryker, 1996; Beaver et al., 2001; Frank et al., 2001). To ensure that the proper statistic was always used in our assessments, we first tested each dataset for normality using the Kolmogorov-Smirnov and equal variance tests (SigmaStat Software; Systat, Richmond, CA). Datasets that passed these tests were then assessed with parametric statistics (Student's $t$ tests for planned comparisons, ANOVAs followed by protected Tukey's tests in all other cases); otherwise, Mann-Whitney $U$ tests were used. ANOVAs or repeated-measure ANOVAs (for hour-by-hour comparisons of post-MD sleep values) followed by Tukey's protected tests were used to evaluate polysomnographic data as described previously (Frank et al., 2001).

\section{Results}

\section{Vigilance states}

As shown in Figure 1, the main groups of cats had similar amounts of REM sleep, NREM sleep, and wake in the baseline, $\mathrm{MD}$, and post-MD periods, demonstrating that the cats were at similar developmental stages with respect to sleep/wake organization, that all cats were mostly awake during the MD period and 


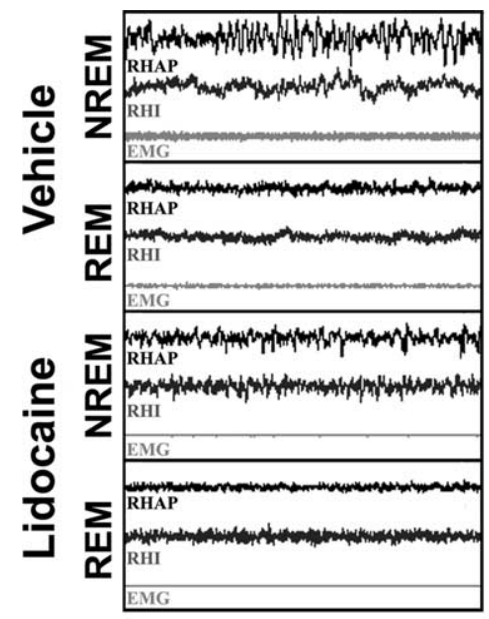

Baseline

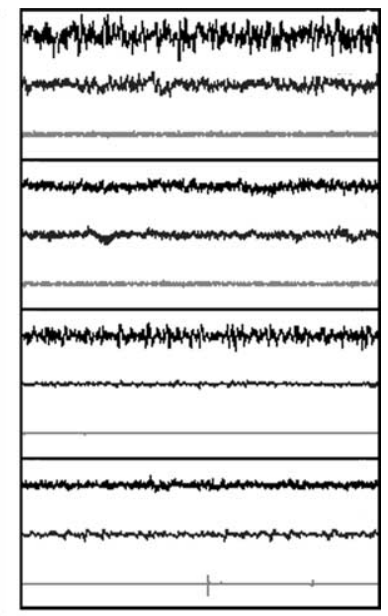

Infusion
Figure 2. EEG and EMG polygraphic traces from cats representative of the vehicle- and lidocaine-infused groups. Top panels represent REM sleep and NREM sleep EEGs from anterior positions far from the infusion site [right hemisphere anterior position (RHAP)], within $1 \mathrm{~mm}$ of the infusing cannula in visual cortex [right hemisphere infusion (RHI)] and the nuchal EMG in a control cat infused bilaterally with artificial CSF (vehicle). Bottom panels represent equivalent measurements made from an experimental cat infused bilaterally with lidocaine. Note the pronounced suppression of EEG activity in V1 in the lidocaine cat during the infusion period in both REM and NREM sleep. Each panel represents $30 \mathrm{~s}$.

mostly asleep during the post-MD period, and that sleep was not grossly perturbed by lidocaine infusions. Sleep and wake amounts in all groups were comparable with normative values from non-infused cats undergoing the same MD procedures (Frank et al., 2001). There were no significant differences in wake, REM sleep, or NREM sleep amounts in the three main groups of kittens during the baseline, $\mathrm{MD}$, or post-MD periods (ANOVA baseline: wake, $F=1.15, p=0.337$; NRM, $F=0.476, p=0.628$; REM, $F=1.02, p=0.381$; MD period: wake, $F=0.875, p=$ 0.433; post-MD: wake, $F=0.617, p=0.552$; NREM, $F=0.78$, $p=0.926$; REM, $F=0.121, p=0.887)$. We found a significant increase in NREM sleep amounts in the post-MD period in all cats (baseline vs post-MD, Student's $t$ test: LIDO, $p<0.008$; $\mathrm{VEH}, p<0.002$; SHAM, $p<0.0004)$. There were no other significant changes in sleep values in the post-MD period.

\section{Sleep EEG analysis: visual cortex}

We were not able to analyze V1 EEGs in all cats because EEGs at this site often contained artifacts, possibly attributable to increased movement near the electrodes compared with anterior sites in which the electrodes could be more rigidly fixed. However, we were able to measure V1 EEGs from five LIDO and five VEH cats and from three SHAM cats. As shown in Figure 2, intracortical lidocaine profoundly suppressed EEG activity in REM and NREM sleep. This reduction in cortical EEG activity was highly significant, as measured by spectral analyses of the EEGs (Fig. 3a,b). The ANOVA revealed only main effects of the infusion (no significant time interactions), indicating that the suppression was uniform across the infusion period (REM sleep: delta, $F=63.7, p<0.0001$; sigma, $F=46.4, p<0.0001$; HF, $F=$ 32.5, $p<0.0001$; NREM sleep: delta, $F=76.1, p<0.0001$; sigma, $F=94.06, p<0.0001$; HF: $F=66.7, p<0.0001)$.

\section{Sleep EEG analysis: anterior positions}

Spectral analysis of the frontal/parietal sleep EEGs verified that lidocaine infusions did not grossly alter EEGs distant from the

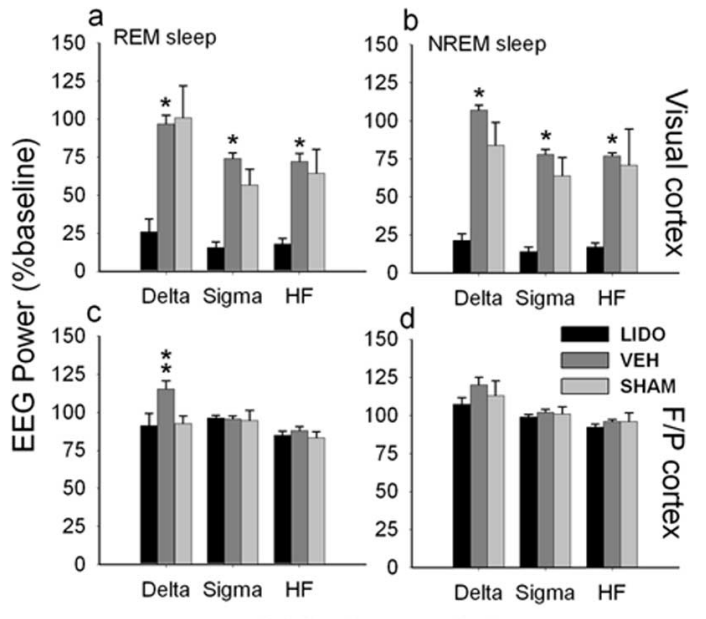

Ad lib sleep period

Figure 3. Mean \pm SEMs of EEG power during post-MD sleep in visual cortex $(\boldsymbol{a}, \boldsymbol{b})$ and frontal/parietal (F/P) cortex $(\boldsymbol{c}, \boldsymbol{d})$ in cats infused intracortically with lidocaine (LID0) or vehicle (VEH) or lidocaine above the pial surface (SHAM). EEG data are averaged into delta $(0.5-4.0 \mathrm{~Hz})$, sigma $(10-15 \mathrm{~Hz}), \operatorname{or~HF}(15-40 \mathrm{~Hz})$ bands and are expressed as a percentage of mean baseline values. Single asterisks indicate significant difference between VEH and LIDO values (ANOVA, $p<0.0001)$. Double asterisks indicate significant difference between VEH and LIDO, SHAM values (ANOVA, $p<0.048$ ).

infusion sites and that all cats were equally alert during the MD. As shown in Figure 3, $c$ and $d$, frontal/parietal EEG activity was comparable across all groups during the infusion period with the exception of a slight reduction in REM delta power in the LIDO and SHAM groups compared with VEH across the ad libitum sleep period (ANOVA; $F=3.325 ; p<0.048$; Tukey's test, $p<$ 0.05 ; no significant time interactions). Spectral analysis of the waking EEGs during the MD period showed no differences between groups (ANOVA; $F=0.875 ; p=0.433$ ) and verified that all cats were alert as wake delta power (expressed as a percentage of baseline wake delta power) did not exceed baseline wake values (means \pm SEMs; LIDO, $88.7 \pm 4.4 \%$; VEH, $93.14 \pm 8.7 \%$; SHAM, $76 \pm 3.8 \%)$.

\section{Optical and microelectrode recordings}

Reversible inactivation of V1 in the post-MD sleep period reduced OD plasticity. Optical maps from a cat with normal vision and cats representative of the three main experimental groups showed a large shift in neuronal responses toward the nondeprived eye only in the VEH and SHAM hemispheres (Fig. 4). This shift in ocular dominance was significantly reduced in hemispheres infused with lidocaine during sleep as measured by mean optical CBIs and MIs (Table 1). Although this effect was overall highly significant across both hemispheres and in hemispheres ipsilateral to the deprived eye, MI scores in hemispheres contralateral to the deprived eye were slightly more variable and did not reach significance.

We also found that optical CBIs from cortical regions $>3 \mathrm{~mm}$ from the cannula site in lidocaine-infused hemispheres showed large shifts toward the nondeprived eye that were similar in magnitude to optical CBIs from vehicle-infused hemispheres [means \pm SEMs; lidocaine, $>3 \mathrm{~mm}$ (seven hemispheres), $0.73 \pm$ 0.01 ; vehicle (seven hemispheres), $0.72 \pm 0.02$ ] and significantly more shifted than areas near the cannula site (Student's $t$ test, $p<$ 0.001). A similar gradient in MI scores was also observed (data not shown).

The optical data were verified by microelectrode recordings, 


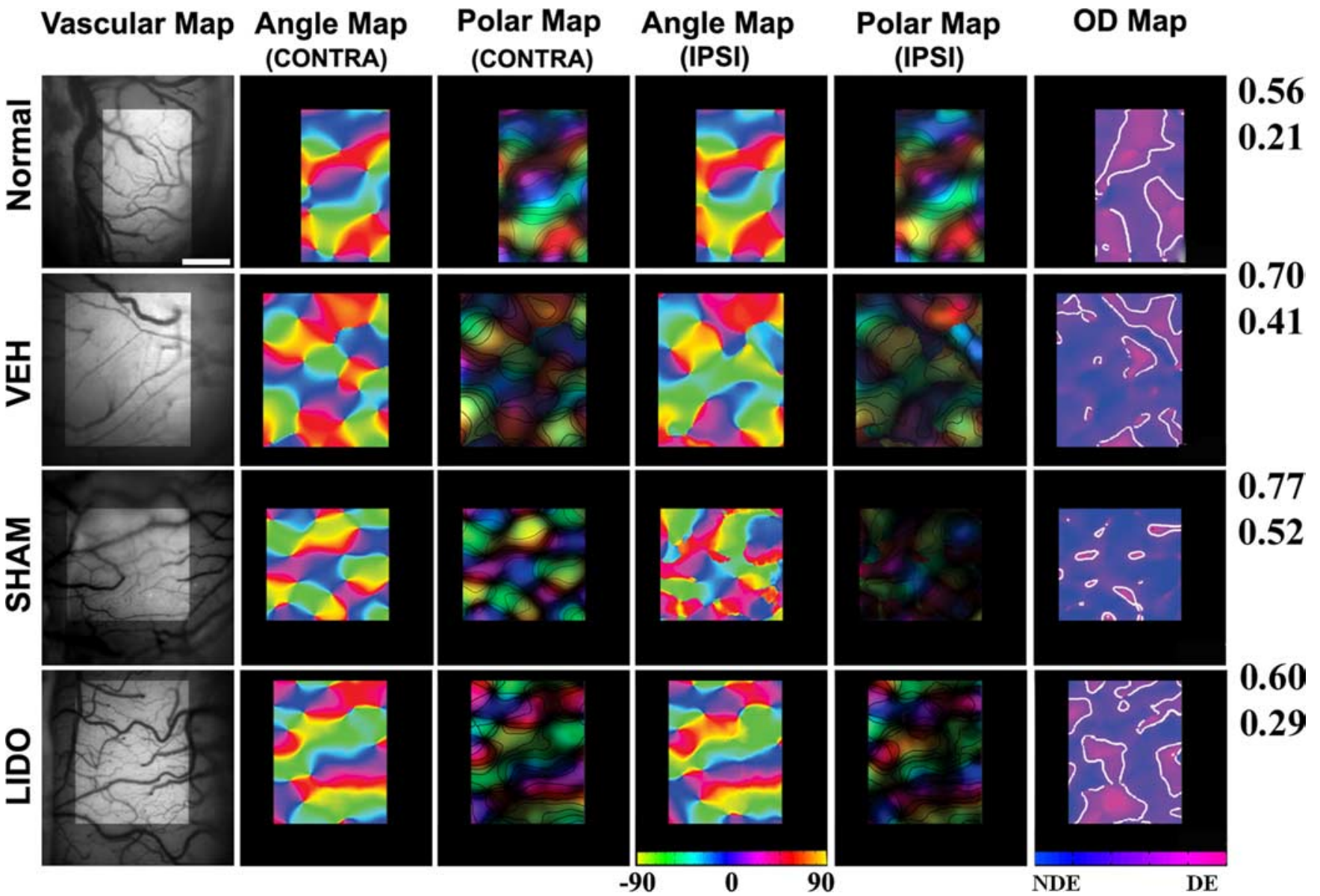

Figure 4. Optical maps in representative animals from each of the main experimental groups and from a normally sighted, critical-period cat. Maps are from hemispheres ipsilateral to the right (deprived) eye. The first column shows maps of surface vasculature above primary visual cortex. The inset area corresponds to the cortical region that was optimally focused. The second and third and the fourth and fifth columns show angle and polar maps from the same regions after nondeprived [contralateral (CONTRA)] and deprived [ipsilateral (IPSI)] eye stimulation. For both angle and polar maps, the color of each pixel represents the orientation of the optimal stimulus (key to color assignment is given in inset bar). For polar maps, the brightness represents the magnitude of the response driven by either eye. Contour lines in the polar maps are provided to highlight pinwheel centers and radiating orientation lines. The sixth column represents $O D$ maps for the deprived eye (DE; magenta) and nondeprived eye (NDE; blue). Contour lines demarcate regions preferentially activated by the deprived eye, and values to the right are corresponding optical CBI (top) and MI (bottom) values (for details, see Materials and Methods for details). Note that deprived-eye angle and polar maps are more degraded compared with nondeprived eye maps in the VEH and SHAM animals and less degraded in the LIDO animal. There is a corresponding greater loss of deprived-eye territories in the SHAM and VEH animals compared with the LIDO animal. Scale bar, $1 \mathrm{~mm}$.

Table 1. Scalar measures of ocular dominance and monocularity in optical maps of visual cortex

\begin{tabular}{llll}
\hline Group & Number of hemispheres & Optical CBI & Optical MI \\
\hline VEH all & $12(2)$ & $0.70 \pm 0.01$ & $0.43 \pm 0.03$ \\
IPSI & $6(1)$ & $0.72 \pm 01$ & $0.46 \pm 0.02$ \\
CONTRA & $6(1)$ & $0.32 \pm 0.02$ & $0.40 \pm 0.05$ \\
LID0 all & $11(1)$ & $\mathbf{0 . 5 5} \pm \mathbf{0 . 0 2}$; ANOVA & $\mathbf{0 . 2 9} \pm \mathbf{0 . 0 1}$; ANOVA \\
& & $F=26.74 ; p<0.001$ & $F=12.1, p<0.01$ \\
& & $p<0.001$ versus VEH & $p<0.001$ versus VEH \\
& & $p<0.001$ versus SHAM & $p<0.001$ versus SHAM \\
IPSI & 6 & $\mathbf{0 . 5 8} \pm \mathbf{0 . 0 1}$ & $\mathbf{0 . 2 9} \pm \mathbf{0 . 0 2}$ \\
& & $p<0.001$ versus VEH & $p<0.001$ versus VEH \\
CONTRA & $5(1)$ & $\mathbf{0 . 5 2} \pm \mathbf{0 . 0 3}$ & $p<0.001$ versus SHAM \\
& & $p<0.001$ versus VEH & $0.28 \pm 0.02$ \\
SHAM all & $10(2)$ & $p<0.01$ versus SHAM & \\
IPSI & 6 & $0.68 \pm 0.02$ & $0.40 \pm 0.03$ \\
CONTRA & $4(2)$ & $0.72 \pm 0.01$ & $0.45 \pm 0.01$ \\
\hline
\end{tabular}

Mean \pm SEM optical CBIs and Mls are shown for all analyzed hemispheres. The number of nonanalyzed, damaged hemispheres is shown in parentheses. A two-way ANOVA was used to evaluate overall significance between groups, with group and hemisphere as factors. Between-group and betweenhemisphere comparisons were made with Tukey's protected $t$ test when significant effects were found in the ANOVA. The values in bold indicate significant differences between the LIDO group and VEH or SHAM groups. IPSI refers to hemispheres ipsilateral to deprived eye; CONTRA refers to hemispheres contralateral to deprived eye. which also showed a suppression of OD plasticity in hemispheres infused with lidocaine. OD histograms and scalar measurements of eye preference calculated for all infused hemispheres showed a pronounced shift toward the open eye in VEH and SHAM-infused hemispheres (Fig. 5, Table 2). However, neurons in hemispheres reversibly inactivated during sleep were significantly less shifted toward the open eye. As was the case for optical recordings, although OD plasticity was reduced in both hemispheres, this effect was more consistent in hemispheres ipsilateral to the deprived eye.

The hemisphere differences appeared to be attributable to greater variability in the effects of MD in hemispheres contralateral to the deprived eye. For example, as shown in Tables 1 and 2, the SEs for optical and microelectrode measurements were generally larger in the contralateral 

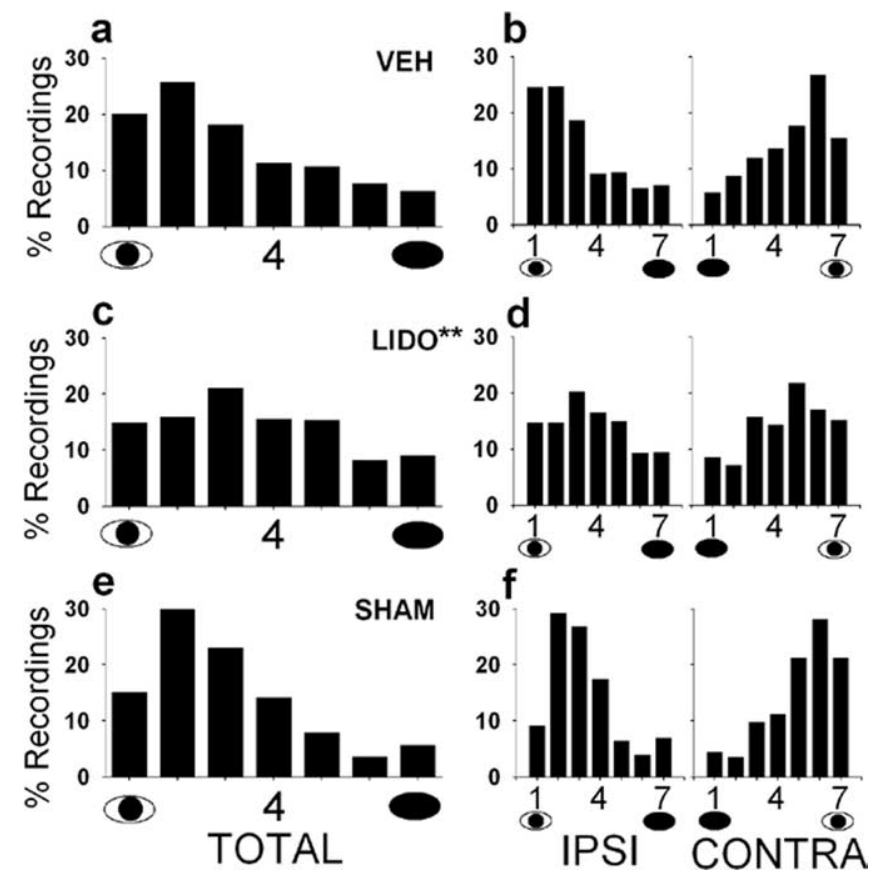

Figure 5. Microelectrode recordings of ocular dominance plasticity in main groups. $\boldsymbol{a}-\boldsymbol{f}$, Ocular dominance histograms compiled from pooled unit recordings in both hemispheres (TOTAL) and in hemispheres ipsilateral (IPSI) and contralateral (CONTRA) to the deprived eye for VEH $(\boldsymbol{a}, \boldsymbol{b}), \operatorname{LIDO}(\boldsymbol{c}, \boldsymbol{d})$, and SHAM $(\boldsymbol{e}, \boldsymbol{f})$ groups. Total histograms are ranked such that 1 represents cells exclusively driven by the nondeprived eye, 7 represents cells driven exclusively by the deprived eye, and 4 represents cells that are driven equally by the two eyes. Histograms for each hemisphere are ranked according to the traditional seven-point scale of Hubel and Wiesel (1970). ${ }^{* *} p<0.05$, significant differences between LIDO and VEH, SHAM distributions (ANOVA) (see Table 2).

hemispheres, and, in three cases (one VEH, two LIDO cats), unit data were excluded from statistical analyses because they were determined to be statistical outliers (values $>3$ SDs from group means). This variability may be related to the slight contralateral bias in kitten V1, which, in our experience, results in more consistent OD shifts in the hemisphere ipsilateral to the deprived eye after short periods of MD.

\section{Effects of lidocaine infusion on visual processing}

The reduction in OD plasticity in lidocaine-infused hemispheres was not attributable to gross abnormalities in visual processing. Nondeprived eye responses from lidocaine-infused hemispheres were robust and selective for orientation as indicated by strong polar maps [which display the orientation selectivity and magnitude of the cortical response (Fig. 4)]. In addition, neurons from lidocaine-infused hemispheres did not differ from SHAM or VEH neurons on any measure of responsiveness other than ocular dominance and monocularity (Table 2). This is consistent with the effects of lidocaine, which dissipate after 45-60 min.

\section{Rescue experiments}

Additional sleep after the lidocaine infusion did not rescue OD plasticity. Mean \pm SEM sleep and wake amounts (as a percentage of total recording time) in the RESCUE cats were comparable with values obtained in the other groups (baseline: wake, $32.8 \pm$ 7.4\%; NREM, $39.31 \pm 3.3 \%$; REM, $27.9 \pm 3.97 \%$; MD period: wake, $94.1 \pm 0.2 \%$; post-MD: wake, $5.9 \pm 0.6 \%$; NREM, $52 \pm$ $4.2 \%$; REM, $41.9 \pm 3.62 \%$ ), and the cats spent most of their time asleep during the additional ad libitum sleep period (NREM, $48 \pm 4.1 \%$; REM, $43.3 \pm 3.7 \%$ ). However, the shift toward the nondeprived eye was reduced in RESCUE cats (Fig. 6). Mean \pm SEM optical CBIs and MIs in three hemispheres (two ipsilateral and one contralateral to deprived eye; one hemisphere could not be analyzed attributable to cortical damage near the infusion site) were comparable with values from the LIDO group $(0.49 \pm 0.02$ and $0.30 \pm 0.5)$. Similar results were obtained with microelectrode recordings, which showed no shift toward the nondeprived eye [CBI and MI \pm SEMs: overall, four hemispheres $(n=532)$, $0.49 \pm 0.02,0.54 \pm 0.01$; two ipsilateral $(n=288), 0.46 \pm 0.04$, $0.52 \pm 0.04$; two contralateral $(n=244), 0.52 \pm 0.04,0.56 \pm$ $0.01]$. These findings demonstrated that the effects of sleep on OD plasticity were time dependent and could be irreversibly disrupted when the cortex was silenced shortly after visual experience.

\section{Discussion}

We investigated the role of cortical activity during sleep in OD plasticity and found that reversibly inactivating the sleeping visual cortex inhibited experience-dependent remodeling in cortical circuits. We also observed that additional sleep after a period of cortical inactivation did not rescue cortical plasticity. These findings demonstrate that neuronal activity during sleep immediately after waking experience is required for the consolidation of OD plasticity.

Our results are consistent with a previous study showing an important role for sleep in OD plasticity. In this previous investigation, we found that $6 \mathrm{~h}$ of sleep after MD enhanced the shift in neuronal responses toward the nondeprived eye, but this enhancement did not occur in cats kept awake in the dark (Frank et al., 2001). In agreement with these results, optical CBIs in sleeping SHAM and VEH cats were comparable with values previously reported after sleep, and cortical inactivation during sleep reduced OD plasticity to levels similar to those observed after sleep deprivation.

Some differences between the two studies include the observation that microelectrode measurements of the shift in neuronal response toward the nondeprived eye in SHAM and VEH cats were slightly lower than values previously reported for sleeping cats (Frank et al., 2001). In addition, sleep deprivation in the dark appeared to be slightly more effective in restoring binocularity after MD than cortical inactivation during sleep (Frank et al., 2001). These small differences, however, may result from a number of factors, including different electrophysiological measurements used in the two studies (i.e., multiunit vs single-unit, unit sampling differences) and the fact that cortical inactivation was $<100 \%$ in the present study (Fig. 3a,b).

Our results are also in agreement with previous investigations showing that continuous blockade of cortical activity (Reiter et al., 1986) or systemic administration of anesthetics after MD (Rauschecker and Hahn, 1987) blocks OD plasticity. In the former study, however, the inactivation occurred during MD and was not restricted to sleep, and, in the latter study, the anesthetic effects were not restricted to $\mathrm{V} 1$ or to a particular vigilance state. In contrast, we now report that cortical inactivation of $\mathrm{V} 1$ during sleep is sufficient to inhibit synaptic remodeling elicited by previous waking experience.

\section{Nonspecific effects of cortical inactivation}

The inhibition of OD plasticity was not attributable to disruption of ongoing sleep behavior or nonspecific effects of the inactivating agent diffusing into the CSF. Although there was a slight reduction in REM sleep EEG delta power in the LIDO and SHAM cats, the amount of sleep and wake in all groups were similar at all 
Table 2. Scalar measures of single-unit ocular dominance and neuronal response properties in VEH-, LIDO-, and SHAM-infused hemispheres

\begin{tabular}{|c|c|c|c|c|c|c|c|}
\hline & $\begin{array}{l}\text { Number of hemispheres; } \\
\text { cells; } \% \text { NR }\end{array}$ & CBI & Ml & $R_{\mathrm{pf}}$ & $R_{\mathrm{VR}}$ & $L_{\mathrm{pf}}$ & $L_{\mathrm{VR}}$ \\
\hline VEH all & $12(1) ; 1558 ; 4.7 \%$ & $0.66 \pm 0.02$ & $0.61 \pm 0.02$ & $3.43 \pm 0.56$ & $0.22 \pm 0.031$ & $4.46 \pm 0.74$ & $0.18 \pm 0.044$ \\
\hline IPSI & $7 ; 835$ & $0.69 \pm 0.01$ & $0.66 \pm 0.02$ & $2.86 \pm 0.80$ & $0.21 \pm 0.06$ & $3.82 \pm 1.1$ & $0.20 \pm 0.09$ \\
\hline CONTRA & $5(1) ; 723$ & $0.38 \pm 0.02$ & $0.55 \pm 0.03$ & $4.0 \pm 0.80$ & $0.23 \pm 0.02$ & $5.10 \pm 1.1$ & $0.17 \pm 0.02$ \\
\hline LIDO all & $10(2) ; 1628 ; 3.6 \%$ & $\begin{array}{c}\mathbf{0 . 5 7} \pm \mathbf{0 . 0 1} ; \text { ANOVA; } \\
F=10.8, p<0.001 ; \\
p<0.004 \text { versus VEH; } \\
p<0.002 \text { versus SHAM }\end{array}$ & $\begin{array}{c}\mathbf{0 . 5 2} \pm \mathbf{0 . 0 3} ; \text { ANOVA; } \\
F=4.4, p<0.02 ; \\
p<0.04 \text { versus VEH }\end{array}$ & $5.83 \pm 1.64$ & $0.24 \pm 0.02$ & $6.48 \pm 1.51$ & $0.21 \pm 0.02$ \\
\hline IPSI & $6 ; 785$ & $\begin{array}{l}\mathbf{0 . 5 6} \pm \mathbf{0 . 0 2} \\
p<0.001 \text { versus VEH; } \\
p<0.05 \text { versus SHAM }\end{array}$ & $0.51 \pm 0.04$ & $6.83 \pm 2.83$ & $0.27 \pm 0.01$ & $7.34 \pm 2.54$ & $0.24 \pm 0.05$ \\
\hline CONTRA & $4(2) ; 843$ & $\begin{array}{l}0.42 \pm 0.01(0.49) \\
p<0.02 \text { versus SHAM }\end{array}$ & $0.53 \pm 0.04(0.56)$ & $4.59 \pm 1.35$ & $0.22 \pm 0.05$ & $5.42 \pm 1.53$ & $0.18 \pm 0.05$ \\
\hline SHAM all & $9(1) ; 1203 ; 2 \%$ & $0.68 \pm 0.02$ & $0.53 \pm 0.03$ & $3.67 \pm 0.20$ & $0.27 \pm 0.03$ & $4.86 \pm 0.38$ & $0.20 \pm 0.03$ \\
\hline IPSI & $4(1) ; 400$ & $0.64 \pm 0.03$ & $0.50 \pm 0.04$ & $3.54 \pm 0.23$ & $0.27 \pm 0.05$ & $4.98 \pm 0.65$ & $0.19 \pm 0.04$ \\
\hline CONTRA & $5 ; 617$ & $0.31 \pm 0.03$ & $0.56 \pm 0.05$ & $3.80 \pm 0.93$ & $0.27 \pm 0.06$ & $4.74 \pm 1.11$ & $0.20 \pm 0.04$ \\
\hline
\end{tabular}

The number of hemispheres (plus nonanalyzed outliers), neurons, and percentage of nonresponsive neurons (\% NR) is shown, along with mean \pm SEM scalar measures of CBI and MI. IPSI refers to hemispheres ipsilateral to deprived eye; CONTRA refers to hemispheres contralateral to deprived eye. A two-way ANOVA was used to evaluate overall significance between groups, with group and hemisphere as factors. Between-group and between-hemisphere comparisons were made with Tukey's protected $t$ test when significant effects were found in the ANOVA. The values in bold indicate significant differences between the LIDO group and VEH or SHAM groups.

a CBI: 0.52, MI: 0.24
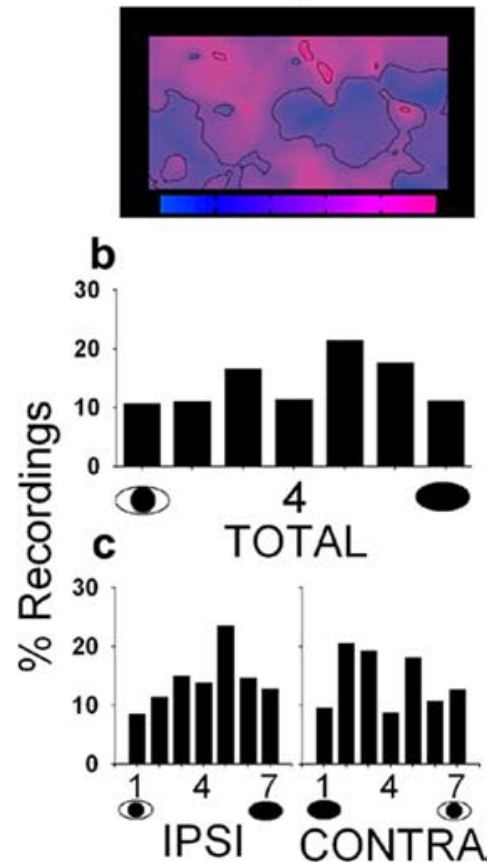

Figure 6. Representative optical data and microelectrode recordings of ocular dominance plasticity in RESCUE cats. $\boldsymbol{a}$, Optical OD ratio map (ipsilateral to the deprived eye) from a representative cat and the corresponding optical CBI and MI values (area represents $\approx 1.5 \times 2 \mathrm{~mm}$ of cortical surface). Color assignments are as in Figure 4. b, $\boldsymbol{c}, 0$ cular dominance histograms for TOTAL ipsilateral (IPSI) and contralateral (CONTRA) hemispheres compiled and ranked as described in Figure 5.

phases of the experiment. It is unlikely that this modest change in EEG activity played a significant role in our results. SHAM cats showed the same EEG changes yet demonstrated robust shifts in neuronal responses toward the nondeprived eye, as measured by optical and unit CBIs (Tables 1,2).

Our results were also not attributable to a general loss of visual responsiveness or other gross abnormalities in neurons transiently silenced during sleep. First, the recovery from cortical inactivation occurred under anesthesia, which blocks further re- modeling in visual cortex (Freeman, 1979; Imamura and Kasamatsu, 1991). Consequently, the inhibition of OD plasticity in the LIDO cats cannot be ascribed to any transient changes in neuronal activity that might have occurred during recovery (e.g., rebound excitation). Second, optical maps generated by nondeprived eye stimulation in lidocaine-infused hemispheres were always strong and comparable with nondeprived eye responses from VEH and SHAM-infused cats (Fig. 4). Furthermore, microelectrode recordings showed no significant differences in visual responses other than ocular dominance and monocularity between VEH, LIDO, and SHAM cats (Table 2). For these reasons, we conclude that the reduction of plasticity observed in LIDO cats was specifically attributable to the inactivation of V1 during sleep.

\section{Mechanisms of sleep-dependent synaptic plasticity}

A number of potential mechanisms have been proposed to explain the beneficial effects of sleep on learning and memory and, by extension, synaptic plasticity. For example, gene expression, protein synthesis, intrinsic neuronal activity, and changes in neurotransmitter or hormone release have all been hypothesized to play important roles in sleep-dependent synaptic remodeling (Sejnowski and Destexhe, 2000; Benington and Frank, 2003; Steriade and Timofeev, 2003; Gais and Born, 2004; Walker and Stickgold, 2004). In general, these putative mechanisms can be broadly divided into those that are activity dependent and those that involve processes that do not require neuronal activity. For example, sleep is associated with hormonal changes [e.g., cortisol suppression and growth hormone release (Cauter and Spiegel, 1999)] that may influence synaptic plasticity (Benington and Frank, 2003; Gais and Born, 2004). The release of these hormones, however, is unlikely to require cortical activity. Therefore, a logical first step in identifying the underlying mechanisms governing sleep-dependent plasticity is to determine whether this process is activity dependent.

Our findings show that cortical activity during sleep is an essential component of sleep-dependent plasticity during the critical period for visual development. They thus narrow the range of possible candidate mechanisms to those that are activity dependent. One possible mechanism may be a reactivation during sleep of the imbalanced activity in the two visual pathways 
triggered by MD. Reactivations of waking neural activity during sleep or sleep-like neuronal events (e.g., during quiet arousal in the rat) have been reported in the hippocampus and somatosensory cortex (Wilson and McNaughton, 1994; Kudrimoti et al., 1999; Louie and Wilson, 2001; Hoffman and McNaughton, 2002), and similar replay in V1, if present, might consolidate synaptic changes elicited during wake. Alternatively, it is possible that, during sleep, there are changes in intrinsic activity within remodeling circuits that play a crucial role in synaptic modification (Benington and Frank, 2003; Tononi and Cirelli, 2003). It has been proposed, for example, that the periodic influxes of calcium that occur during oscillatory neuronal bursting promote the expression of plasticityrelated genes or proteins (Sejnowski and Destexhe, 2000; Benington and Frank, 2003). It is also possible that the EEG slow waves of NREM sleep downregulate certain synapses and/or promote long-term depression (LTD) (Benington and Frank, 2003; Tononi and Cirelli, 2003). Interestingly, LTD appears to mediate the rapid loss of function in deprived-eye visual pathways after MD (Heynen et al., 2003). Our findings are consistent with both broad categories of hypotheses, but, because lidocaine inhibits synaptic and intrinsic action potentials, the relative contribution of network-level and intrinsic forms of activity to sleep-dependent plasticity could not be determined in this study.

\section{Concluding remarks}

Our results raise a number of interesting questions. First, is cortical activity in REM sleep, NREM sleep, or both sleep states necessary for sleep-dependent plasticity? Our experimental design resulted in the silencing of neurons in both REM and NREM sleep, and, although our previous findings indicate that OD plasticity is enhanced by NREM sleep (Frank et al., 2001), REM sleep may also play an important role. Second, is the source of this neuronal activity intracortical or thalamocortical? Both thalamic input and corticocortical interactions govern the rapid changes in cortical circuitry elicited by short-term MD (Trachtenberg et al., 2000). Sleep provides a rich source of intracortical and thalamocortical activity (Benington and Frank, 2003; Steriade and Timofeev, 2003), but only one of these forms of neural activation may be required for sleep-dependent plasticity. Finally, what cellular and molecular mechanisms of plasticity are triggered by the activity of the sleeping brain? Several activitydependent components of OD plasticity have been identified, including NMDA and GABA receptors, neurotrophins, and cAMP-mediated kinase activity (Roberts et al., 1998; Daw et al., 1999; Pizzorusso et al., 2000; Beaver et al., 2001; Hensch, 2003; Liu et al., 2003). Are these also important components of sleep-dependent plasticity? Answers to these questions will help solve the mystery of sleep function and provide a more complete understanding of how experience and sleep shape the brain.

\section{References}

Beaver CJ, Ji Q, Fischer QS, Daw WD (2001) Cyclic AMP-dependent protein kinase mediates ocular dominance shifts in cat visual cortex. Nat Neurosci 4:159-163.

Benington JH, Frank MG (2003) Cellular and molecular connections between sleep and synaptic plasticity. Prog Neurobiol 69:77-101.

Cauter EV, Spiegel K (1999) Circadian and sleep control of hormonal secre- tions. In: Regulation of sleep and circadian rhythms (Zee PC, Turek FW, eds), pp 397-425. New York: Dekker.

Cirelli C, Gutierrez CM, Tononi G (2004) Extensive and divergent effects of sleep and wakefulness on brain gene expression. Neuron 41:35-43.

Crair MC, Gillespie DC, Stryker MP (1998) The role of visual experience in the development of columns in cat visual cortex. Science 279:566-570.

Datta S, Mavanji V, Ulloor J, Patterson EH (2004) Activation of phasic pontine-wave generator prevents rapid eye movement sleep deprivationinduced learning impairment in the rat: a mechanism for sleepdependent plasticity. J Neurosci 24:1416-1427.

Daw NW, Gordon B, Fox KD, Flavin HJ, Kirsch JD, Beaver CJ, Ji Q-H, Reid SNM, Czepita D (1999) Injection of MK-801 affects ocular dominance shifts more than visual activity. J Neurophysiol 81:204-215.

Frank MG, Issa NP, Stryker MP (2001) Sleep enhances plasticity in the developing visual cortex. Neuron 30:275-287.

Freeman RD (1979) Effects of brief uniocular "patching" on kitten visual cortex. Trans Ophthalmol Soc U K 99:382-385.

Gais S, Born J (2004) Declarative memory consolidation: Mechanisms acting during human sleep. Learn Mem 11:679-685.

Gordon JA, Stryker MP (1996) Experience-dependent plasticity of binocular responses in the primary visual cortex of the mouse. J Neurosci $16: 3274-3286$

Hensch TK (2003) Controlling the critical period. Neurosci Res 47:17-22.

Heynen AJ, Yoon BJ, Liu CH, Chung HJ, Huganir RL, Bear MF (2003) Molecular mechanism for loss of visual cortical responsiveness following brief monocular deprivation. Nat Neurosci 6:854-862.

Hoffman KL, McNaughton BL (2002) Coordinated reactivation of distributed memory traces in primate cortex. Science 297:2070-2073.

Hubel DH, Wiesel TN (1970) The period of susceptibility to the physiological effects of unilateral eye closure in kittens. J Physiol (Lond) 206:419-436.

Huber R, Ghilardi MF, Massimini M, Tononi G (2004) Local sleep and learning. Nature 430:78-81.

Hupe J-M, Chouvet G, Bullier J (1999) Spatial and temporal parameters of cortical inactivation by GABA. J Neurosci Methods 86:129-143.

Imamura K, Kasamatsu T (1991) Ocular dominance plasticity restored by NA infusion to aplastic visual cortex of anesthetized and paralyzed kittens. Exp Brain Res 87:309-318.

Issa NP, Trachtenberg JT, Chapman B, Zahs KR, Stryker MP (1999) The critical period for ocular dominance plasticity in the ferret's visual cortex. J Neurosci 19:6955-6978.

Jones BE, Law J, Griffin G, Coleman T, Jha S, Frank MG (2004) Sleep dependent plasticity requires cortical activity. Soc Neurosci Abstr 30:77.3.

Kalatsky VA, Stryker MP (2003) New paradigm for optical imaging: temporally encoded maps of intrinsic signal. Neuron 38:529-545.

Kudrimoti HS, Barnes CA, McNaughton BL (1999) Reactivation of hippocampal cell assemblies: effects of behavioral state, experience and EEG dynamics. J Neurosci 19:4090-4101.

Laguzzi RF, Adrien J, Bourgoin S, Hamon M (1979) Effects of intraventricular injection of 6-hydroxydopamine in the developing kitten. I. On the sleep waking cycles. Brain Res 160:445-459.

Laureys S, Peigneux P, Phillips C, Fuchs S, Degueldre C, Aerts J, Del Fiore G, Petiau C, Luxen A, Van der Linden M (2001) Experience-dependent changes in cerebral functional connectivity during human rapid eye movement sleep. Neuroscience 105:521-525.

Liu S, Rao Y, Daw N (2003) Roles of protein kinase A and protein kinase G in synaptic plasticity in the visual cortex. Cereb Cortex 13:864-869.

Louie K, Wilson MA (2001) Temporally structured replay of awake hippocampal ensemble activity during rapid eye movement sleep. Neuron 29:145-156.

Malpeli JG (1999) Reversible inactivation of subcortical sites by drug injection. J Neurosci Methods 86:119-128.

Nadasky Z, Hirase H, Czurko A, Csicsvari J, Buzsaki G (1999) Replay and time compression of recurring spike sequences in the hippocampus. J Neurosci 19:9497-9507.

Pizzorusso T, Fagiolini M, Gianfranceschi L, Porciatti V, Maffei L (2000) Role of neurotrophins in the development and plasticity of the visual system: experiments on dark rearing. Int J Psychophysiol 35:189-196.

Rauschecker JP, Hahn S (1987) Ketamine-xylazine anesthesia blocks consolidation of ocular dominance changes in kitten visual cortex. Nature 326:183-185. 
Reiter HO, Waitzman DM, Stryker MP (1986) Cortical activity blockade prevents ocular dominance plasticity in the kitten visual cortex. Exp Brain Res 65:182-188.

Ribeiro S, Mello CV, Velho T, Gardner TJ, Jarvis ED, Pavlides C (2002) Induction of hippocampal long-term potentiation during waking leads to increased extrahippocampal zif-268 expression during ensuing rapideye-movement sleep. J Neurosci 22:10914-10923.

Roberts EB, Meredith MA, Ramoa AS (1998) Suppression of NMDA receptor function using antisense DNA block ocular dominance plasticity while preserving visual responses. J Neurophysiol 80:1021-1032.

Sejnowski TJ, Destexhe A (2000) Why do we sleep? Brain Res 886:208-223.

Siegel JM (2001) The REM sleep-memory consolidation hypothesis. Science 294:1058-1063.
Steriade M, Timofeev I (2003) Neuronal plasticity in thalamocortical networks during sleep and waking oscillations. Neuron 37:563-576.

Tononi G, Cirelli C (2003) Sleep and synaptic homeostasis: a hypothesis. Brain Res Bull 62:143-150.

Trachtenberg JT, Trepel C, Stryker MP (2000) Rapid extragranular plasticity in the absence of thalamocortical plasticity in the developing primary visual cortex. Science 287:2029-2032.

Vertes RP, Eastman KE (2000) The case against memory consolidation in REM sleep. Behav Brain Sci 23:867-876.

Walker MP, Stickgold R (2004) Sleep-dependent learning and memory consolidation. Neuron 44:121-133.

Wilson MA, McNaughton BL (1994) Reactivation of hippocampal ensemble memories during sleep. Science 265:676-682. 